\title{
A rare case report of thymolipoma in a 4-year-old child
}

\author{
Bhushan M. Warpe \\ ${ }^{1}$ (Pathology Department, IGGMC, Nagpur city, Maharashtra state, MUHS Nashik university, India.)
}

\begin{abstract}
Thymolipoma is a rare benign tumor of the anterior mediastinum with mean age of incidence of 20 to 30 years with rare occurrence in children. All clinicians are not aware of this entity and so the diagnosis is easily missed. It is defined as a mass forming lesion composed of circumscribed mature adipose tissue and unremarkable thymus. I present a case of 4-year-old child with history of breathlessness. USG chest of the child had revealed a space occupying mass in the anterior mediastinum of $5 \times 6 \times 4 \mathrm{cms}$. On resection, the $550 \mathrm{gm}$ weight mass showed capsulated, soft to firm, yellowish-tan colored areas. Sections from the mass showed predominantly adipose tissue containing interconnecting cords of thymus. Although rare, thymolipoma must be considered in the differential diagnosis of anterior mediastinal tumors.
\end{abstract}

Keywords: Anterior mediastinal tumor, Mediastinum, Thymolipoma.

\section{Introduction}

Thymolipoma is an uncommon benign tumor of the anterior mediastinum. ${ }^{[1]}$ The cause is disputed, but the most accepted theory of pathogenesis is replacement of the thymus by mature adipose tissue. The tumor grows slowly and can become large. Although most patients with a thymolipoma are asymptomatic, local nonspecific symptoms may present or the tumor may be associated with parathymic and autoimmune syndromes such as myasthenia gravis, hyperthyroidism, lymphangiomas, aplastic anemia, chronic lymphocyte leukemia, and Hodgkin disease.$^{[2]}$ The tumor may resemble cardiomegaly in a chest $\mathrm{x}$-ray, an anterior mediastinal mass on USG chest and is difficult to distinguish from other mediastinal tumors. We describe a 4 year-old child case who presented with progressive breathlessness since last one year, but the definitive diagnosis was thymolipoma.

\section{Case Details}

A 4-year-old child with history of progressive breathlessness since last one year was brought by parents and admitted in our hospital. No other associated symptoms of fever, cough, cold were noted in the child.

The only finding of interest in the physical examination was the complete absence of vesicular breath sounds in the left side of the chest. The laboratory findings were normal.

Simple posteroanterior chest $\mathrm{x}$-ray identified a large mass in the left side of the chest, located in the pleural cavity and anterior mediastinum. The initial suspected diagnosis was cardiomegaly. To rule out cardiomegaly, USG chest was done which showed an anterior mediatinal mass of $5 \times 6 \times 4 \mathrm{cms}$ that occupied the entire left side of the chest. The mass had laterally shifted the mediastinum and collapsed the lung in the uppermost region of this side of the chest. No signs of invasion of neighboring structures were observed.

FNAC done under radiological guidance revealed lymphocytes and fragments of mature adipose tissue, without any thymic epithelial element. The differential diagnoses were lipoma, thymolipoma and liposarcoma. To relive of the respiratory distress, the patient underwent surgery in which the tumor was resected through a left anterolateral thoracotomy. The soft to firm mass measured $5 \times 6 \times 4 \mathrm{~cm}$ and weighed 550 gms weight. It was easily delineated from the surrounding tissue by a thin capsule and removed easily. It resembled lipoma on gross examination. The cut section showed yellowish-tan colored areas (Fig.1- inset). 


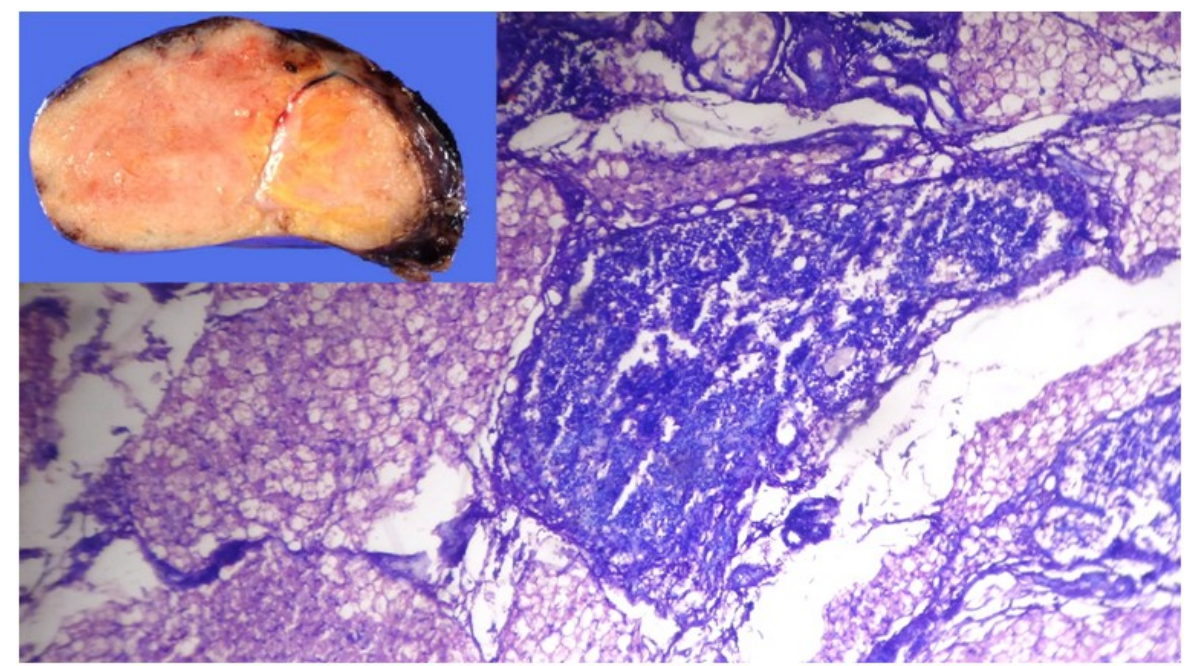

Figure 1: Microphotograph : Thymic mass section shows lobules of mature adipose tissue intimately associated with unremarkable thymic tissue. (H\&E, x100) Inset shows cut section of thymolipoma with soft to firm, yellowish-tan colored areas.

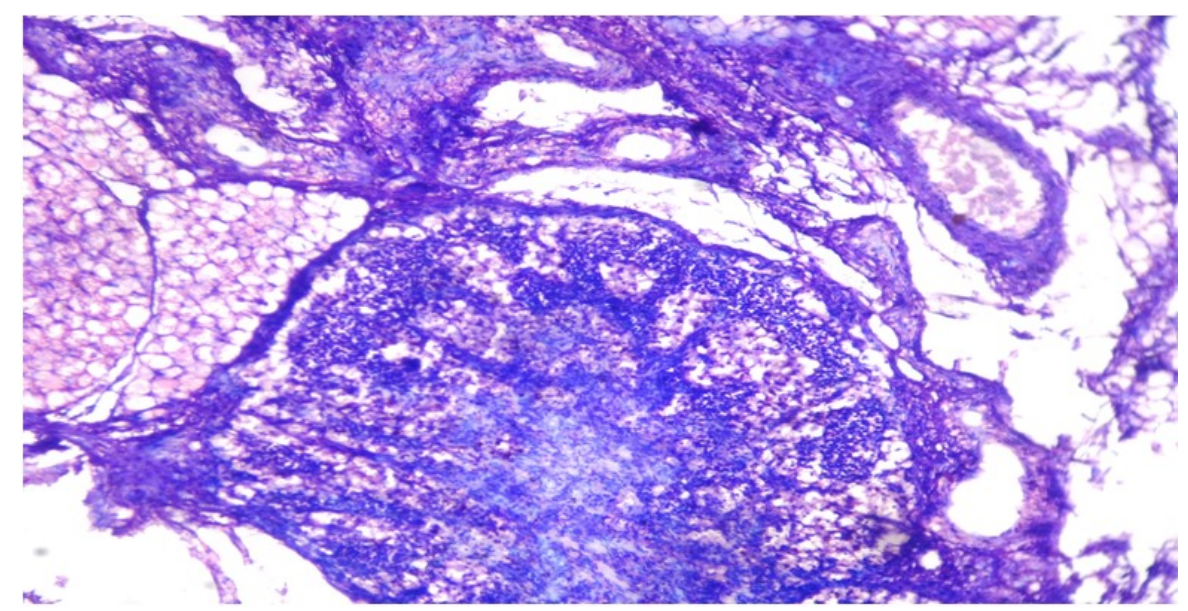

Figure 2: Microphotograph : Thymic mass section shows lobules of mature adipose tissue intimately associated with unremarkable thymic tissue. (H\&E, x400)

Pathological examination of the resected block showed a thin fibrous capsule surrounding lobules of mature adipose tissue intimately associated with unremarkable thymic tissue containing cortex, medulla and Hassall's corpuscles. Thus based on interconnecting cords of thymic epithelial cells and groups of adipocytes set in a loose fibrous component, the diagnosis of thymolipoma was made. (Figs.1\&2) Differential diagnosis to thymolipoma is limited. Lipoma is ruled out by the presence of the thymic component in our case while atrophic thymus with adipose replacement is an incidental finding and does not form a mass.

The patient's recovery was satisfactory and he was discharged 14 days after the operation.

\section{Discussion}

Thymolipoma is an uncommon benign neoplasm that accounts for $2 \%$ to $9 \%$ of thymic tumors. ${ }^{[3]}$ The incidence is the same in men and women and onset can occur at any age but preferably in 20 to 30 years of age and rarely seen in children. According to our review of the literature, its incidence is approximately 0.12 cases per 100000 inhabitants per year. ${ }^{[4]}$

Several theories of the pathogenesis of the tumor of thymolipoma have been proposed. It is also known as lipoma of the thymus, benign thymoma, thymolipomatous hamartoma, lipothymoma, and mediastinal lipoma because the tumor grows as multifocal benign proliferation of perivascular connective tissue which starts in the medulla. In the process of growth, the fatty tissue pulls small particles of thymic tissue into its substance. This explains the presence of two components of different origins in one tumor. Thus the replacement of diffuse thymic hyperplasia by adipose tissue seems to be the most widely accepted explanation. ${ }^{[5]}$

Thymolipoma may present as an asymptomatic tumor that does not affect adjacent structures, and so large sizes upto $20 \mathrm{cms}$ on average can be reached before diagnosis. When such tumors grow in the left side of 
the chest, images may resemble cardiomegaly, as occurred in our reported case. Approximately half of the patients with this tumor experience symptoms such as dyspnea, chest pain and weight loss, ${ }^{[4]}$ and myasthenia gravis is present in up to $10 \%$ of the cases. Other related autoimmune diseases are systemic erythematous lupus (SLE),

The weight of these tumors, according to our review of the literature, ranges from $154 \mathrm{~g}$ to $6000 \mathrm{~g}^{[7]}$; in our case, the tumor weighed more than $550 \mathrm{~g}$, and measured $5 \times 6 \times 4 \mathrm{~cm}$. Thymolipomas are characterized by mesodermal (fat) and endodermal (thymic epithelium) elements, as we saw in our case. They appear as lobulated well-encapsulated tumors with septal divisions. Large lobes of mature adipose tissue are interspersed with small areas of thymic tissue. ${ }^{[8]}$ These tumors are often confused with cardiomegaly in a simple $\mathrm{x}$-ray, as happened in our case. ${ }^{[9]}$ Although diagnosis can be established by imaging, to differentiate thymolipoma from lipoma, thymic hyperplasia, and liposarcoma; a biopsy might be needed to confirm the histology of the tumor. The only curative treatment for this benign tumor with excellent prognosis is surgical resection of the tumor.

\section{Conclusion}

Thymolipoma is a rare anterior mediastinal tumor, more so in children. Its composed of a mixture of thymic elements and adipose tissue on histopathology. It is associated with autoimmune disorders and is benign tumor with excellent prognosis on complete resection. Though radiological investigations can be helpful to define the size, location and extent of the tumor, histopathology is the gold standard test that gives the most definitive diagnosis of thymolipoma. Despite its rarity and difficulty of pre-operative diagnosis, it must be included in the differential diagnoses of anterior mediastinal tumors.

\section{Acknowledgements}

I acknowledge the love and support of my family and friends ('apane potte' in hindi) for making this article-a reality.

\section{References}

[1]. Tindall H, Tandon AP, Ionescu MI. An unusual case of chest pain and cardiomegaly. Br J Clin Pract. 1990;44:766-7.

[2]. Mc Manus KG, Allen MS, Trastek VF. Lipothymoma with red cell aplasia, hypogammaglobinemia and lichen planus. Ann Thorac Surg. 1994;58:1534-6.

[3]. Fenniche S, Maalej S, Hassene H. Unusual presentation of giant thymolipoma. Tunis Med. 2003;81:59-62.

[4]. Gamondes JP, Balawi A, Greenland T. Seventeen years of surgicaltreatment of thymoma: factors influencing survival. Eur J Cardiothorac Surg. 1991;5:124-31.

[5]. Argani P, Rosai J. Thymoma arising with a thymolipoma. Histopathology. 1998;32:5568-78.

[6]. Wang Y, Sun Y, Zhang J. Diagnosis, treatment and prognosis of thymoma: an analysis 116 cases. Chin Med J. 2003;116:1187-90.

[7]. Benton C, Gerard P. Thymolipoma in a patient with Graves' disease: case report and review of the literature. J Thoracic Cardiovasc Surg. 1996;51:428.

[8]. Shields TW, Robinson PG. Mesenchymal tumors of the mediastinum. In: Shields TW, editor. General thoracic surgery.Vol. 2. Philadelphia: 2000; p. 2357-423.

[9]. Yeh H, Gordon A, Kirschner PA, et al. Computed tomography and sonography of thymolipoma. AJR Am J Roentgenol. 1983;140:1131. 